\title{
Effect of Waste Plastic as Bitumen Modified in Asphalt Mixture
}

\author{
Mohd Ezree Abdullah ${ }^{1}$, Siti Aminah Abd Kader ${ }^{2}$, Ramadhansyah Putra Jaya ${ }^{3,4,}$, Haryati \\ Yaacob $^{5}$, Norhidayah Abdul Hassan ${ }^{6}$, and Che Norazman Che Wan ${ }^{7}$ \\ ${ }^{1}$ Faculty of Civil and Environmental Engineering, Universiti Tun Hussein Onn Malaysia, 86400 Batu \\ Pahat, Johor Bahru, Malaysia \\ ${ }^{2,3,5,6}$ Faculty of Civil Engineering, Department of Geotechnics and Transportation, Universiti \\ Teknologi Malaysia, 81310 UTM Skudai, Johor Bahru, Malaysia \\ ${ }^{4}$ Faculty of Engineering, Department of Civil and Environmental Engineering, Universiti Teknologi \\ PETRONAS, 32610 Seri Iskandar, Perak Darul Ridzuan, Malaysia \\ ${ }^{7}$ Department of Civil Engineering, Politeknik Ungku Omar, 31400 Ipoh, Malaysia
}

\begin{abstract}
The objectives of this study are to investigate the engineering properties of the asphalt mixtures containing waste plastic at different percentages i.e. $4 \%, 6 \%, 8 \%$, and $10 \%$ by weight of bitumen. The experimental tests performed in the study were stability, tensile strength, resilient modulus and dynamic creep test. Results showed that the mixture with $4 \%$ plastic has the highest stability $(184 \mathrm{kN})$. However, the stability slightly decreases with the increase of plastic additive. On the other hand, the highest tensile strength among the modified asphaltic concrete is $1049 \mathrm{kPa}$ ( $8 \%$ plastic added). The modified asphalt mixture with $8 \%$ plastic has the highest resilient modulus, which is $3422 \mathrm{MPa}\left(25^{\circ} \mathrm{C}\right)$ and $494 \mathrm{Mpa}$ $\left(40^{\circ} \mathrm{C}\right)$. Where the highest creep modulus recorded is $73.30 \mathrm{Mpa}$ at $8 \%$ plastic added. It can be concluded that the addition of $8 \%$ plastic gave the highest value properties of asphalt mixture. Finally, it can be said that $8 \%$ plastic is the optimum value adding.
\end{abstract}

\section{Introduction}

Plastic is used regularly in today's lifestyle, and its disposal is a great problem. Plastic is a non-biodegradable product; thus, it causes environmental pollution and problems. The issue now is to reduce plastic waste by transforming it into something beneficial. Given that the plastics used in PET bottles, disposal glasses, handbags, and covers of various appliances soften up at $160^{\circ} \mathrm{C}$, a solution is to use waste plastic in such a manner that it gets coated over by an aggregate via heating $\left(140-160^{\circ} \mathrm{C}\right)$. Plastic bags are made from one of three basic types of polyethylene (PE), that is, high-density polyethylene (HDPE), low density polyethylene (LDPE), and linear low-density polyethylene (LLDPE). The thick, silky shopping bags from malls are LLDPE; grocery bags are HDPE; and clothing bags from the dry cleaners are LDPE. Many types of PE are known, with most having the chemical

*Corresponding author: ramadhansyah@utm.my 
formula $\left(\mathrm{C}_{2} \mathrm{H}_{4}\right)$ n. Thus, PE is usually a mixture of similar organic compounds that differ in terms of the value of $n$. One of the main problems of polyethylene is that without special treatment it is not readily biodegradable and thus accumulates. Over 500 billion to 1 trillion plastic bags are consumed worldwide. Plastic waste is a serious problem that can lead to many other problems. It may be hard to totally remove plastic bag usage from our life. Nevertheless, we can manage it by following the common simple rule of $3 R$ : reduce, reuse, and recycle. A recent study had found one more way to reduce the amount of plastic waste by using it in asphalt mixtures [1]. Waste plastic bags can be used as a modifier of hot-mix asphalt [2]. We review the possibility of incorporating waste plastic to improve the performance of asphalt mix. Köfteci [3] reported that utilization of waste plastic in flexible pavement has been established since 2012. Arabani and Pedram [4] reported that modifying the rheological properties of bitumen by mixing with synthetic polymers, such as rubber and plastics, is a common method to improve bitumen quality. Polymer-modified bitumen has better resistance to temperature and water. According to Naskara et. al [5], recycled waste plastics bags in bituminous mix by using $1.2-3 \mathrm{~mm}$ particle size in the surface and base mix design of up to $2.5 \%$ for improved performance of roads to improve the desired mechanical characteristics of a particular road mix. As the result, the stability and flow were improved. Using waste plastics (polyethylene terephalate) in asphalt concrete mixture can replace the mineral coarse aggregate of an equal size $(2.36-4.75 \mathrm{~mm})$ and resulted in an optimum value of $6.6 \%$ vol. This method led also to a reduction of bulk compacted mix density. Modification of asphalt with polymers is one of the best options to improve asphalt properties [6]. The added polymer can strongly improve the binder properties, provide safer road, and reduce the maintenance cost by increasing the stiffness of the bitumen and by improving its temperature susceptibility [7]. Modarres and Hamedi [8] reported that the use of recycled waste plastic in pavement asphalt represents a valuable outlet for such materials. The use of modified bitumen with the addition of processed waste plastic of about $5 \%-10 \%$ by weight of bitumen helps in substantially improving the Marshall stability, fatigue, life strength, and other required properties of bituminous concrete mix, which improves the durability and pavement performance with marginal saving in bitumen usage. The process is environment friendly. The use of waste plastics in the manufacture of roads and laminated roofing also helps to consume large quantity of waste plastics. Thus, these processes are socially highly relevant and give better infrastructure.

\section{Materials and methods}

\subsection{Binder, aggregate and gradation}

The binder used in this study was 60/70 PEN bitumen supplied by Chevron Malaysia. On the other hand, granite aggregates was used throughout this investigation. Prior to aggregate batching, the aggregates were washed, dried and sieved into their respective size ranges. The aggregate gradation conformed to JKR/SPJ/2008-S4 [9] specifications for asphaltic concrete as shown in Fig. 1. 


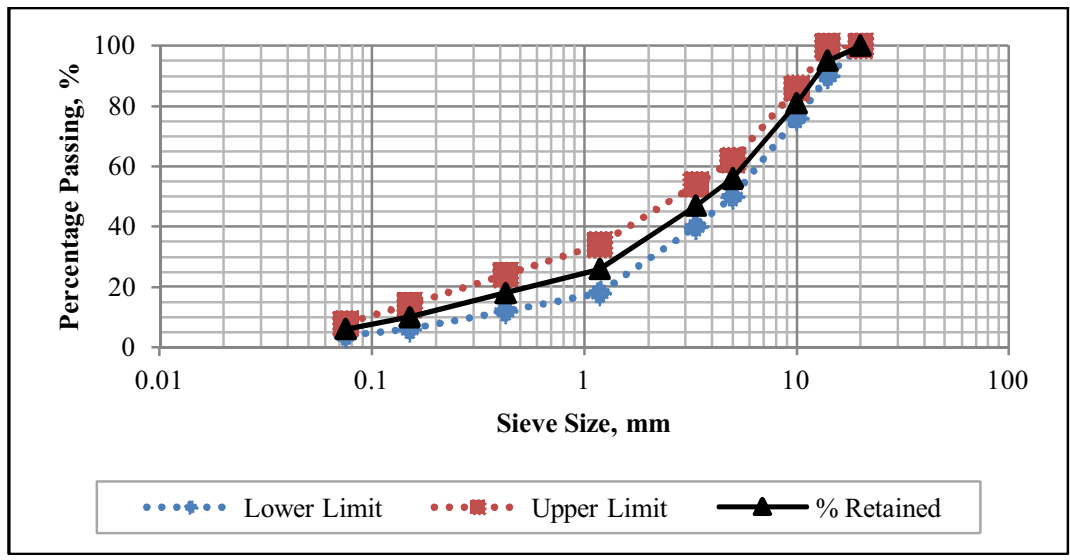

Fig. 1. Aggregate gradation of asphalt mix [9]

\subsection{Mix preparation}

Ordinary Portland cement and hydrated lime were used as filler in this investigation. The $60 / 70$ bitumen was used as binder for mix preparation. The aggregates were first mixed into batches according to the designated gradations and weight. These batches were then heated in an oven at the designated mixing temperature for at least 4 hours before the mixing process. The mixing and compaction temperatures adopted were $180 \pm 0.5^{\circ} \mathrm{C}$. The heated aggregate batches were then mixed with a specified amount of bitumen. The mixes were compacted with 75 blows on each side with the standard Marshall hammer. After compaction, the specimens were removed from the molds and allowed to cool overnight.

\subsection{Marshall stability test}

In the stability test, the specimens were prepared with the specified temperature by immersing in a water bath at a temperature of $60^{\circ} \mathrm{C} \pm 1^{\circ} \mathrm{C}$ for a period of 45 minutes. It was then placed in the Marshall Stability testing machine and loaded at a constant rate of deformation of $50.8 \mathrm{~mm} /$ minute until the maximum load was reached. Test was conducted in accordance with ASTM D6927-06 [10]. The stability result was recorded on the Marshall testing machine in $\mathrm{kN}$.

\subsection{Indirect tensile strength test}

The indirect tensile strength of bituminous mixtures is conducted by loading a cylindrical specimen across its vertical diametral plane at a specified rate of deformation and test temperature. The samples were conditioned at $25^{\circ} \mathrm{C}$ for $2 \mathrm{~h}$. Afterward, diametral compressive loading was applied to the conditioned samples until failure occurred. The peak load at failure is recorded and used to calculate the IDT strength of the specimen. This test followed ASTM D6931-12 [11].

\subsection{Resilient modulus test}

A five pulse indirect tensile modulus test conforming to ASTM D4123 [12] test method was conducted using the Universal Testing Machine, MATTA. The specimen was tested at 
$25^{\circ} \mathrm{C}$ and $40^{\circ} \mathrm{C}$ after a 4-hour-conditioning period. In the test, a pulsed diametral loading force was applied to a specimen and the resulting total recoverable diametral strain was then measured. The test sequence consisted of the application of 150 conditioning pulses followed by 5 pulses where data acquisition took place. The test procedure was repeated by orientating the specimen at 90 degree.

\subsection{Dynamic creep test}

Dynamic creep test is the simplest method of assessing the resistance to permanent deformation or rutting. The specimens that were used in indirect dynamic creep test were the Marshall samples, which dimensions were similar to that of the specimen for indirect tensile resilient modulus test. The prepared samples were conditioned at $40^{\circ} \mathrm{C}$ for about $2 \mathrm{~h}$ to $3 \mathrm{~h}$. Repeated load was applied for 3600 cycles for $40 \mathrm{~min}$ to $45 \mathrm{~min}$. Test was conducted in accordance with BS EN 12697-25 [13].

\section{Results and discussion}

\subsection{Stability}

Fig. 2 shows the relationship between stability and percentage of plastic. The stability of $4 \%$ plastic is the highest (i.e., $184 \mathrm{kN}$ ), and it slightly decreases with the increase of plastic addition. A large gap between the stability of the conventional asphalt mixture and the plastic modified asphaltic concrete is observed (i.e., more than $100 \%$ ). Stability verifies the performance of the asphalt mixture under loading. The results showed that plastic can increase the stability of the asphalt mixture up to certain percentages and after that it will decrease.

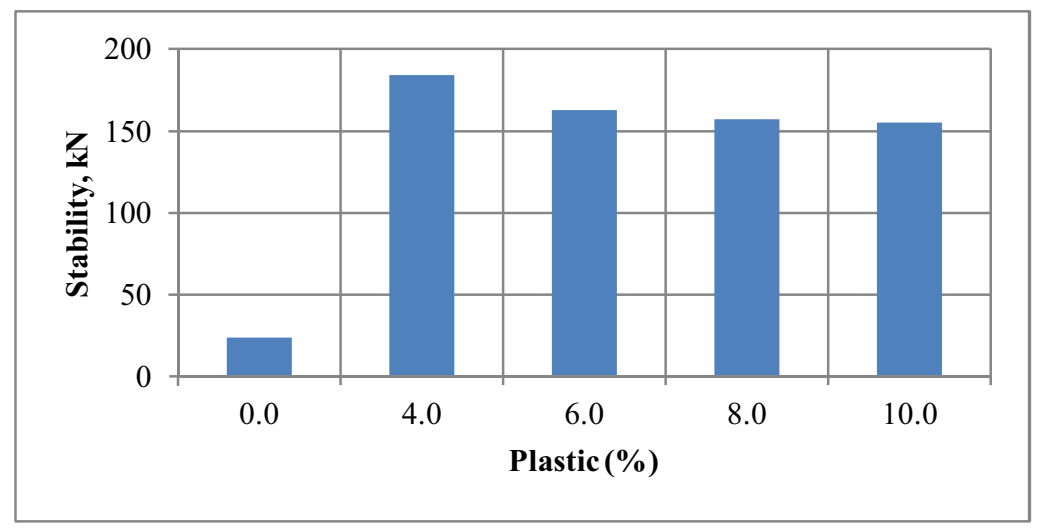

Fig. 2. Effect different percentage plastic to stability

\subsection{Indirect tensile strength}

Indirect tensile strength (ITS) test is used to measure the indirect tensile strength of the mix, which helps to find out the resistance of thermal cracking of the mixture. The ITS results of conventional and modified asphalt mixture are graphically presented in Fig. 3. From the bar chart, the tensile strength of modified asphalt mixture is lower than conventional asphalt mixture. The tensile strength for conventional asphalt mixture is $1095 \mathrm{kPa}$. The highest 
tensile strength among the modified asphaltic concrete is $1049 \mathrm{kPa}$ with $8 \%$ plastic, which is $4 \%$ lower than the conventional asphalt mixture tensile strength. The lowest tensile strength is $844 \mathrm{kPa}$ with $4 \%$ plastic, which is $23 \%$ lower than the conventional asphalt mixture.

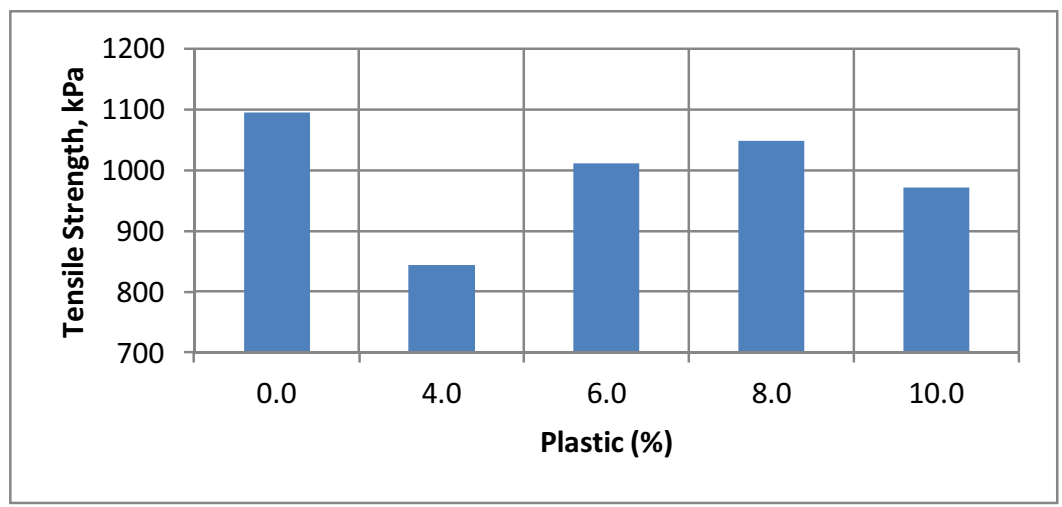

Fig. 3. Effect of different percentage plastic to tensile strength

\subsection{Resilient modulus}

Fig. 4 shows the results of different percentage plastic to resilient modulus at different temperatures. A similar trend is observed for both temperatures. The resilient modulus increases with the increase of the plastic until it reaches the optimum plastic content. The resilient modulus then decreases with further increase of plastic. Modified asphalt mixture with $8 \%$ plastic has the highest resilient modulus for both 25 and $40^{\circ} \mathrm{C}$ temperatures, which is almost double from the conventional asphalt mixture, that is, $3422 \mathrm{MPa}$ for $25^{\circ} \mathrm{C}$ and $494 \mathrm{Mpa}$ for $40^{\circ} \mathrm{C}$. In addition, $4 \%$ plastic has the lowest resilient modulus, that is, $69 \%$ and $51 \%$ lower than the conventional asphalt mixture for 25 and $40^{\circ} \mathrm{C}$ temperatures.

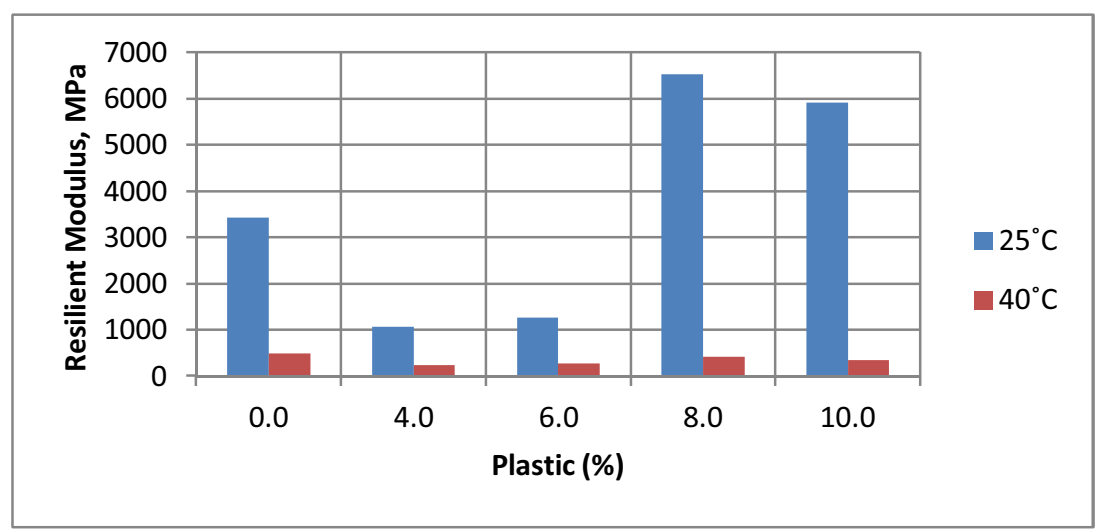

Fig. 4. Effect of different percentage plastic to resilient modulus at $25^{\circ} \mathrm{C}$ and $40^{\circ} \mathrm{C}$

\subsection{Dynamic creep}

The dynamic creep test result is presented in Fig. 5. Dynamic creep test is repeated on the asphaltic concrete and causes the development of rutting. The bar chart in Figure 5 shows that the creep modulus increases with the increased percentage of plastic until $8 \%$ of plastic 
and then decreases. The highest creep modulus is $73.30 \mathrm{Mpa}$ with $8 \%$ of plastic. The lowest creep modulus is $24.92 \mathrm{Mpa}$ with $4 \%$ plastic, which is $5 \%$ lower than the conventional asphalt mixture. Recycling of plastic bags is useful to reduce permanent deformation in the form of rutting and reduce low temperature cracking of pavement surfacing.

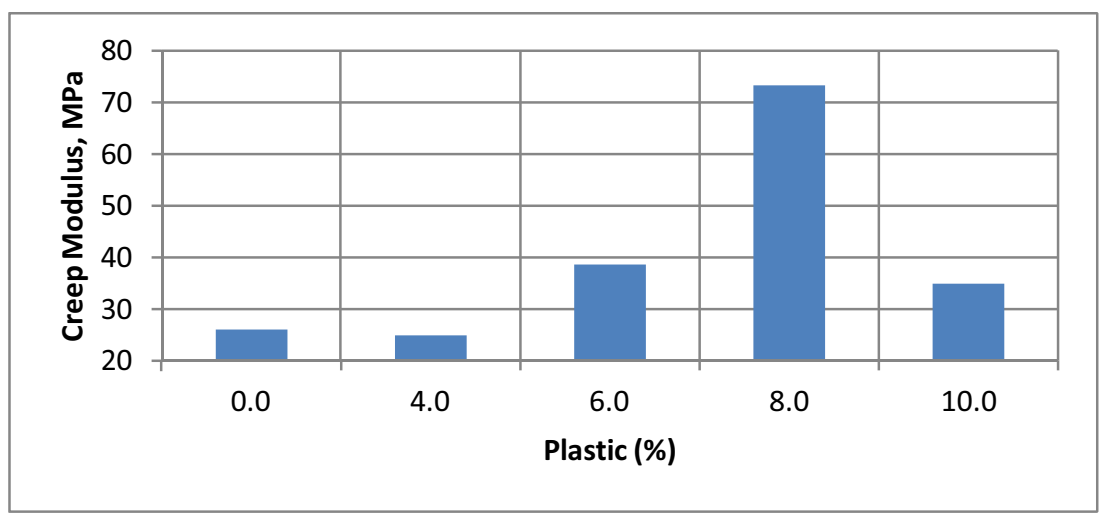

Fig. 5. Effect of different percentage of plastic to creep modulus

\section{Conclusions}

Based on the result and analysis:

(a) The highest tensile strength among the modified asphaltic concrete is $1049 \mathrm{kPa}$ with $8 \%$ plastic added.

(b) Modified asphalt mixture with $8 \%$ plastic added is the highest resilient modulus for both $25^{\circ} \mathrm{C}$ and $40^{\circ} \mathrm{C}$ temperatures which is $3422 \mathrm{MPa}$ for $25^{\circ} \mathrm{C}$ and $494 \mathrm{Mpa}$ for $40^{\circ} \mathrm{C}$. Where the highest creep modulus is $73.301 \mathrm{Mpa}$ with $8 \%$ of plastic added.

(c) Since $8 \%$ plastics added give the highest value for tests, it can be conclude that $8 \%$ plastic added is the optimum value.

Research work presented in this paper was supported by Ministry of Higher Education Malaysia (MOHE), Fundamental Research Grant Scheme (research grant no. 1458), Universiti Tun Hussein Onn Malaysia and Universiti Teknologi Malaysia (research grant no. Q.J130000.2522.11H58), the financial assistance for this study is highly appreciated.

\section{References}

[1] M.R. Mohd Hasan, C. Baron, Y. Zhanping, J. Ali, A.H. Patricia and O.H. Meor, A simple treatment of electronic-waste plastics to produce asphalt binder additives with improved properties, Constr. Build. Mater., 110, 79-88 (2016)

[2] I.M. Khan, K. Shahid, A.A. Majed and F.A. Feras, Asphalt design using recycled plastic and crumb-rubber waste for sustainable pavement construction, Procedia Eng., 145, 1557-1564 (2016)

[3] S.Köfteci, A. Perviz and K. Baurzhan, Performance evaluation of bitumen modified by various types of waste plastics, Constr. Build. Mater., 73, 592-602 (2014)

[4] M. Arabania and M. Pedram, Laboratory investigation of rutting and fatigue in glassphalt containing waste plastic bottles, Constr. Build. Mater., 116, 378-383 (2016) 
[5] M. Naskara, T.K. Chakia and K.S. Reddy, Effect of waste plastic as modifier on thermal stability and degradation kinetics of bitumen/waste plastics blend, Thermochimica Acta, 509(1-2), 128-134 (2010)

[6] N. Abdul Hassan, G.D. Airey, N.I. Md. Yusoff, M.R. Hainin, R. Putrajaya, M.E. Abdullah and M.M.A. Aziz, Microstructural characterisation of dry mixed rubberised asphalt mixtures, Constr. Build. Mater., 82, 173-183 (2015)

[7] M.E. Abdullah, K.A. Zamhari, R. Buhari, M.N. Nayan and M.R. Hainin, Short term and long term aging effects of asphalt binder modified with montmorillonite, Key Eng. Mater., 594-595, 996-1002 (2014)

[8] A. Modarres and H. Hamedi, Effect of waste plastic bottles on the stiffness and fatigue properties of modified asphalt mixes, Mater. Design, 61, 8-15 (2014)

[9] JKR/SPJ/2008-S4, Standard Specification for Road Works Section 4: Flexible Pavement.

[10] ASTM D6927, Standard Test Method for Marshall Stability and Flow of Bituminous Mixtures, Annual Book of ASTM Standards, West Conshohocken, (2006)

[11] ASTM D6931, Standard Test Method for Indirect Tensile (IDT) Strength of Bituminous Mixtures, Annual Book of ASTM Standards, West Conshohocken, (2012)

[12] ASTM D4123, Standard Test Method for Indirect Tension Test for Resilient Modulus of Bituminous Mixtures, Annual Book of ASTM Standards, West Conshohocken, (1995)

[13] BS EN 12697-25, Bituminous Mixtures, Test Methods, Cyclic Compression Test, British Standards Institution, London, (2016) 